\title{
Endoscope-Assisted Trichophytic Anterior Hairline Brow Lift
}

\author{
Ji Min Kim¹, Jeong-Geun Hong ${ }^{2}$ \\ ${ }^{1}$ Plastic and Reconstructive Surgery, \\ College of Medicine, The Catholic \\ University of Korea, Seoul; ${ }^{2}$ Metro Plastic \\ Surgery Clinic, Incheon, Korea
}

Background The brow lift plays an essential role in upper face rejuvenation. The authors designed a new brow lift technique, the endoscope-assisted trichophytic anterior hairline brow lift. It combines the advantages of an endoscopic approach and an anterior hairline approach. This technique was applied to 13 patients with aesthetically excellent results.

Methods A trichophytic incision line was designed within the fine hairs of the entire anterior hairline and the incision was extended to the temporal hair-bearing scalp. After the incision, most of the procedures were conducted in a similar way to the conventional endoscopic brow lift. Without direct visualization, the dissection was extended down onto the root of the nose and the forehead. An endoscope was used from $2 \mathrm{~cm}$ above the supraorbital notch to avoid supraorbital nerve injury. Scars were assessed with the Stony Brook Scar Evaluation Scale (SBSES) at the time the sutures were removed. The visual analogue scale (VAS) score was checked at postoperative 1-year follow-up. The surgical outcomes for brow position and subjective satisfaction were rated with the Global Aesthetic Improvement Scale (GAIS) at postoperative 1-month followup. The dermatome of the deep branch of the supraorbital nerve, especially around the hairline, was checked with a two-point discrimination test.

Results From October 2012 through August 2015, 13 endoscope-assisted trichophytic anterior hairline brow lifts were performed. The GAIS score was 1.62 on average. The VAS score was 2.09 on average. No permanent nerve damage has been reported except for 2 cases of temporary paresthesia. These fully recovered at 1-month follow-up. Conclusions Based on our results, we think our technique could be a safe and effective treatment option for brow ptosis patients with a high hairline.

Keywords Endoscopes, Facelift, Visual analogue scale
No potential conflict of interest relevant to this article was reported. tage of good surgical exposure with magnification, a short incisional scar, and a relatively low risk of scalp denervation. But acquiring the technique requires a long learning period. In addition, in some cases it is difficult to fixate the forehead musculature adequately $[2,3]$, and it may be difficult to avoid widening of the forehead.

The anterior hairline approach is one of the most widely accepted approaches in patients with high hairlines. This technique offers good surgical exposure. Moreover, it makes it possible to lower a high hairline. But it has disadvantages, such as visible scars and scalp denervation $[3,4]$.

The authors designed a new brow lift technique, an endoscopeassisted trichophytic anterior hairline brow lift. This new technique combines the advantages of an endoscopic approach and an anterior hairline approach. It has the advantage of providing good sur- 
gical exposure, adequate surgical fixation, and reduced scalp denervation. Hairline scars can be hidden by a trichophytic incision. This closure allows hair growth through the incision scar $[5,6]$.

This brow lift approach was applied to 13 patients with aesthetically excellent results.

\section{METHODS}

The "trichophytic closure" technique was first introduced by Marzola [6] in 2005. Trichophytic closure allows the growth of hair through the incision scar. Thus, this technique has also been known as "scarless closure". We used this trichophytic incision and closure method to approach brow musculature.

A wavy trichophytic incision was designed within the fine hairs along the entire anterior hairline, and it was extended to the tem-

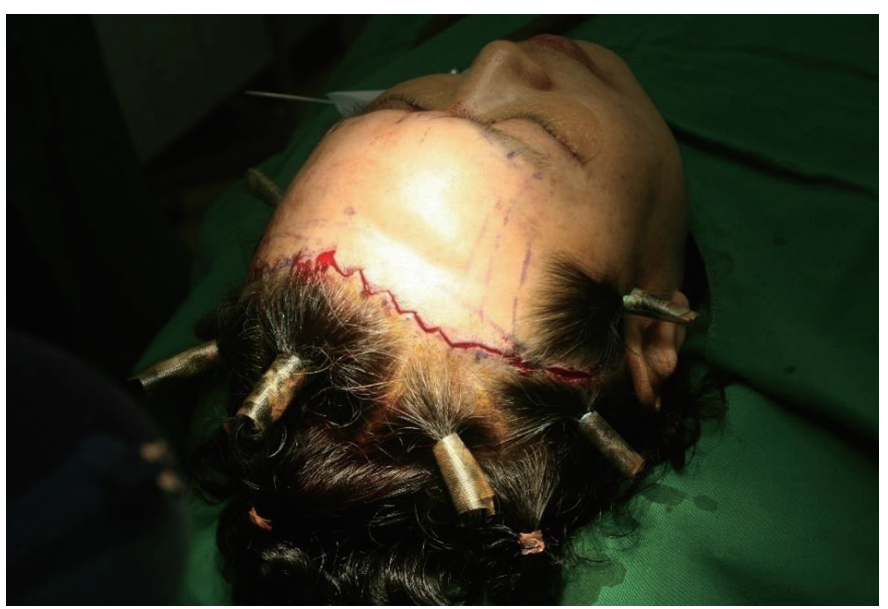

Fig. 1. A trichophytic incision line was designed within the fine hairs along the anterior hairline, with the incision extended along the entire anterior hairline incision to the temporal hair-bearing scalp.

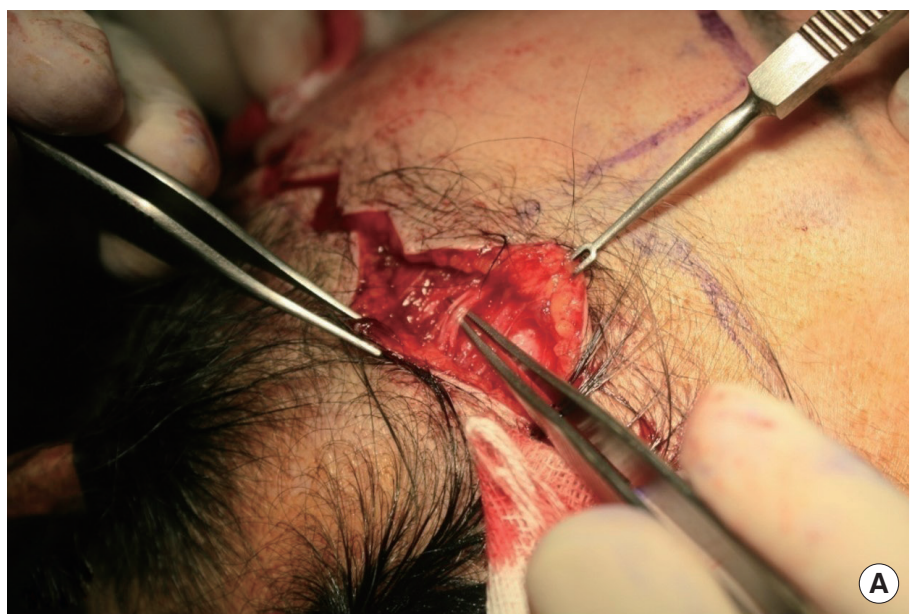

poral area (Fig. 1). The incision was beveled to transect the hair shafts, allowing hair growth through the scar and making future camouflage possible (Fig. 2).

When incising near the temporal fusion lines, 2 -cm-wide subcutaneous dissections were performed to identify and preserve the
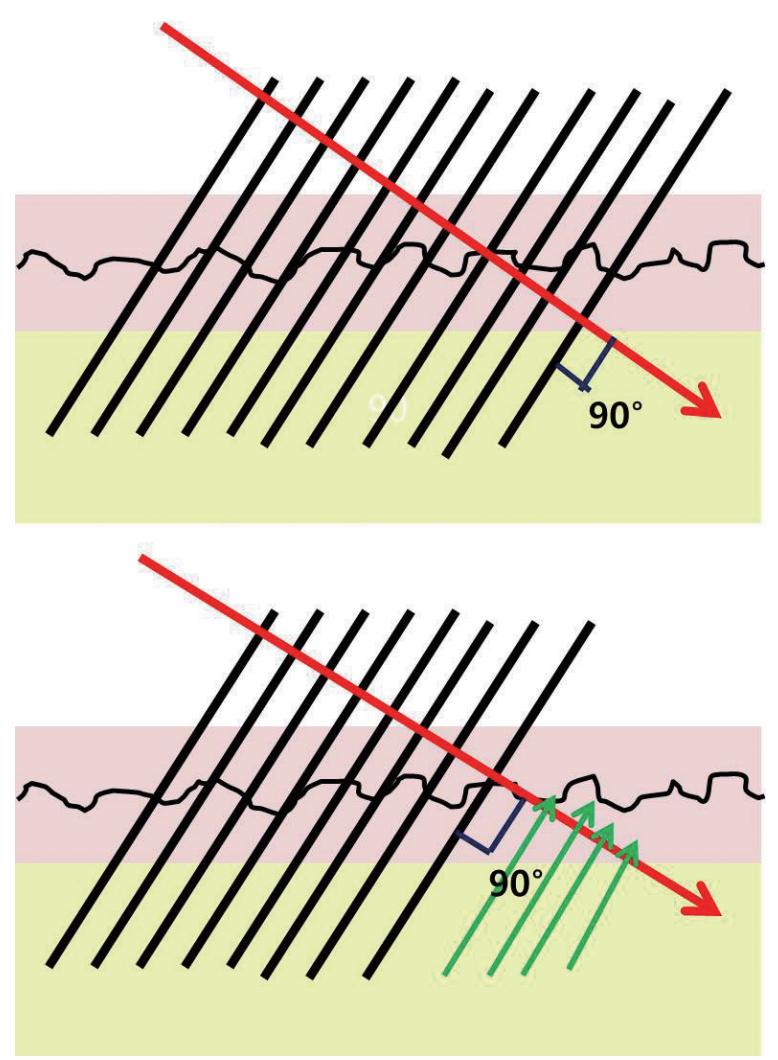

Fig. 2. A beveling incision was made at about $90^{\circ}$ to the hairs, allowing hair growth through the scar that makes future camouflage possible.

Fig. 3. (A) The nerve branches were identified directly and preserved though subcutaneous dissection $2 \mathrm{~cm}$ in width. (B) Subperiosteal dissection was conducted over the vertex posteriorly for lowering a high hairline.

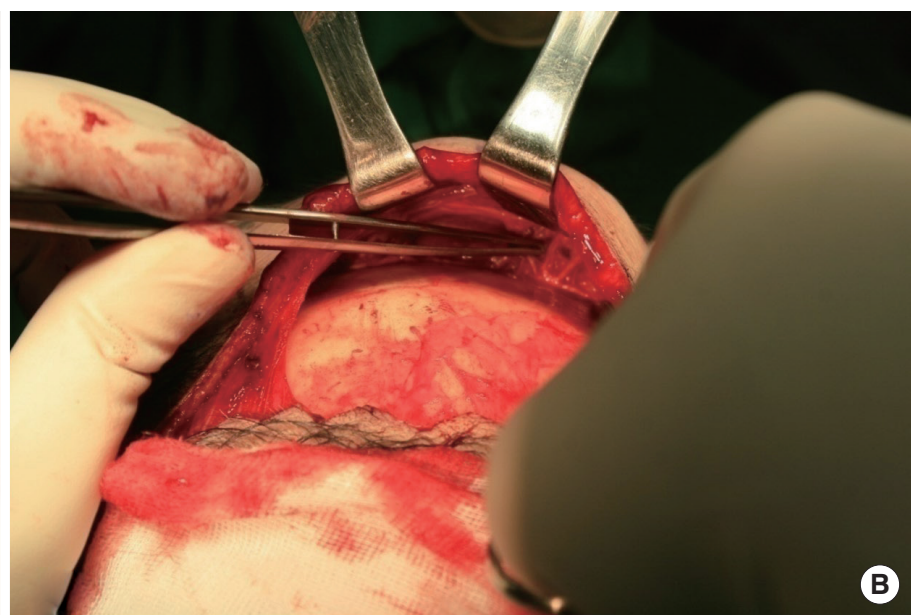


deep branches of the supraorbital nerve (Fig. 3A). Subperiosteal dissection was extended to the vertex to advance the hairline (Fig. 3B).

After incision, the procedures were conducted in a way similar to a conventional endoscopic brow lift. Without direct visualization, the dissection was extended down onto the root of the nose and the forehead. An endoscope was used from $2 \mathrm{~cm}$ above the supraorbital notch to avoid supraorbital nerve injury. The periosteum around the supraorbital rim was divided, and, lateral to the 'zone of fixation', a temporal pocket was made above the deep temporal fascia. The connection between the central and temporal pocket at the temporal fusion line divided in a lateral-to-medial direction. Medial orbital muscles such as corrugator muscles were manipulated. The amount of the skin resection was kept conservative to avoid excessive tension on the wound, and the skin was closed meticulously.

Scars were assessed with the Stony Brook Scar Evaluation Scale (SBSES) at the time of suture removal (Table 1). This scale is the only method that has been adopted by the United States Food and Drug Administration (FDA) as one of the required outcome measurements of wound repair in clinical trials [7]. In addition, the 1-year postoperative visual analogue scale (VAS) score was checked (from 0 , no visible scar, to 10, unacceptable scar).

Patient satisfaction was surveyed and rated with the Global Aesthetic Improvement Scale (GAIS) at 1 month postoperatively (Table 2). A GAIS score of 1 means exceptionally improved; 2, very improved; 3 , improved; 4 , unaltered; and 5 , worsened.

Table 1. Stony Brook Scar Evaluation Scale

\begin{tabular}{lcc}
\hline Category & Response & Score \\
\hline Width & $\geq 2 \mathrm{~mm}$ & 0 \\
& $\leq 2 \mathrm{~mm}$ & 1 \\
Height & Elevated/depressed & 0 \\
& Flat & 1 \\
Color (compared to & Darker (red, purple, brown, & 0 \\
surrounding skin) & or black) & 1 \\
Hatch/suture marks & Same or lighter & 0 \\
& Present & 1 \\
Overall appearance & Absent & 0 \\
& Poor & 1 \\
\hline
\end{tabular}

Dermatome of the deep branch of the supraorbital nerve, especially around the hairline, was checked with a two-point discrimination test.

\section{RESULTS}

From October 2012 to August 2015, endoscope-assisted trichophytic anterior hairline brow lifts were performed in 13 cases (Table 3). The patients were 54.62 years old on average (11 females, 2 males). The average follow-up was 14.38 months postoperatively. The GAIS score was 1.62, which was in the range between 'exceptionally improved' and 'very improved.' This meant that most of the patients were satisfied with the results. The average VAS score was 2.09. This meant that the scars were acceptable to the patients. There were 2 cases of temporary paresthesia, which might have resulted from traction injury during the operation. Sensation was fully recovered after 1 month in both cases. There was no permanent nerve damage. Two patients showed visible scars and under-

Table 3. Patient data

\begin{tabular}{lccccc}
\hline No. & $\begin{array}{c}\text { Sex/Age } \\
\text { (year) }\end{array}$ & $\begin{array}{c}\text { Follow-up period } \\
\text { (month) }\end{array}$ & GAIS & SBSES & VAS \\
\hline 1 & $F / 60$ & 24 & 1 & 4 & 1 \\
2 & $F / 41$ & 13 & 2 & 5 & 1 \\
3 & $F / 49$ & 9 & 1 & 5 & Not yet $^{\text {a) }}$ \\
4 & $F / 48$ & 21 & 1 & 5 & 2 \\
5 & $M / 54$ & 11 & 3 & 3 & 5 \\
6 & $F / 60$ & 14 & 1 & 5 & 1 \\
7 & $F / 51$ & 15 & 2 & 5 & 2 \\
8 & $F / 65$ & 10 & 1 & 4 & $N^{2} \mathrm{et}^{\mathrm{a})}$ \\
9 & $\mathrm{~F} / 44$ & 12 & 1 & 5 & 4 \\
10 & $\mathrm{~F} / 52$ & 14 & 2 & 4 & 1 \\
11 & $\mathrm{M} / 57$ & 13 & 2 & 5 & 3 \\
12 & $\mathrm{~F} / 61$ & 12 & 3 & 5 & 2 \\
13 & $\mathrm{~F} / 68$ & 19 & 1 & 5 & 1 \\
Average & 54.62 & 14.38 & 1.62 & 4.62 & 2.09 \\
\hline
\end{tabular}

${ }^{\text {al }}$ Patients no. 3 and 8 have been followed for 9 months, visual analogue scale score was not checked yet.

F, female; M, male; GAIS, Global Aesthetic Improvement Scale; SBSES, Stony Brook Scar Evaluation Scale; VAS, visual analogue scale.

Table 2. Global Aesthetic Improvement Scale score

\begin{tabular}{|c|c|c|}
\hline & Degree & Description \\
\hline 1 & Exceptional improvement & Excellent corrective result \\
\hline 2 & Very improved patient & Marked improvement of the appearance, but not completely optimal \\
\hline 3 & Improved patient & Improvement of the appearance, better compared with the initial condition, but a touch-up is advised \\
\hline 4 & Unaltered patient & The appearance substantially remains the same compared with the original condition \\
\hline 5 & Worsened patient & The appearance has worsened compared with the original condition \\
\hline
\end{tabular}


went laser treatment. In all cases, hair growth concealed the scar along the hairline.

\section{Case}

A 60-year-old female with the brow ptosis and a drowsy appearance visited our outpatient clinic (Fig. 4A). She underwent an endoscope-assisted trichophytic anterior hairline brow lift. She was satisfied with results at postoperative 1-month follow-up (Fig. 4B). With the help of the trichophytic incision, the grown hair along the incision line concealed the scar 2 years after the operation (Fig. 4C).

Fig. 5 shows preoperative and postoperative clinical photographs of patients who underwent endoscope-assisted trichophytic anterior hairline brow lifts combined with upper or lower blepharoplasty for upper face rejuvenation. A 61-year-old female underwent an endoscope-assisted trichophytic anterior hairline forehead lift with lower blepharoplasty. At postoperative 18 months, the photograph showed satisfying results (Fig. 5A and 5B).

A 52-year-old female underwent an endoscope-assisted tricho-
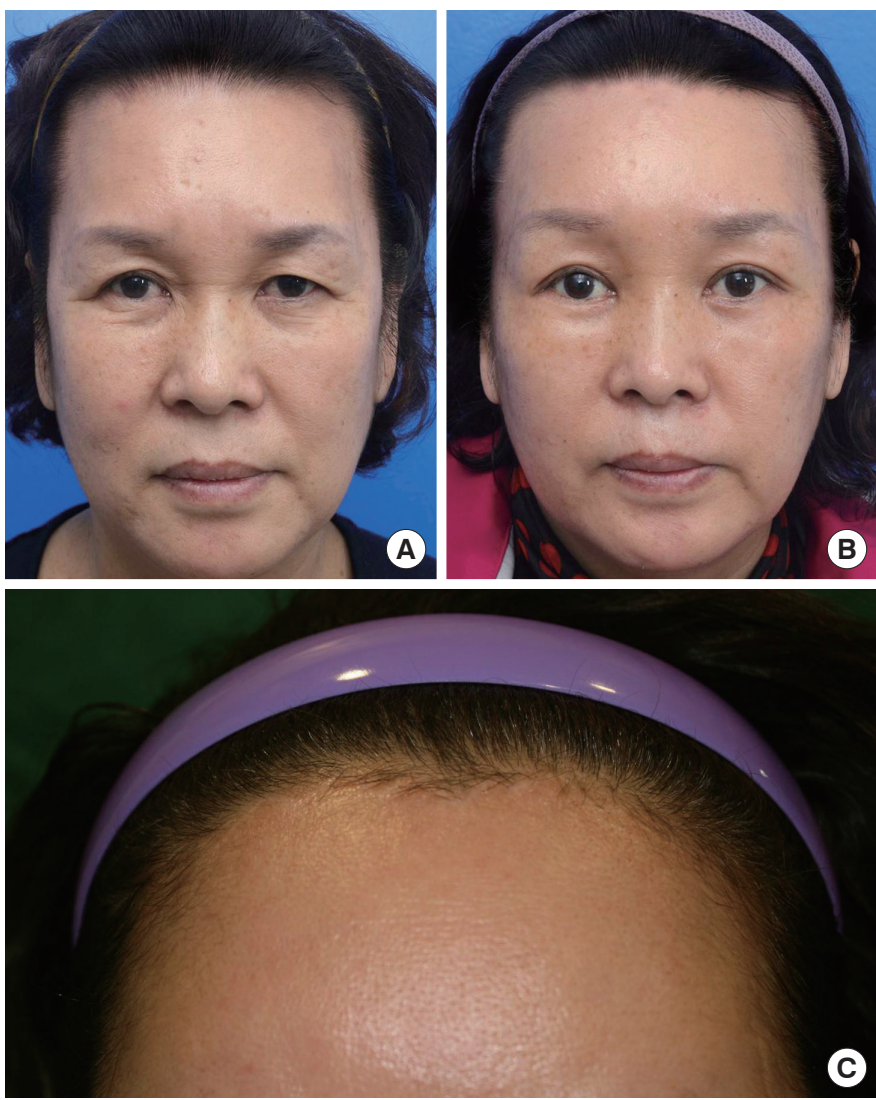

Fig. 4. (A) A 60-year-old female shows brow ptosis on both sides and a drowsy appearance. (B) At 1 month postoperatively from the endoscopic-assisted trichophytic anterior hairline brow lift procedure, she was satisfied with the results. (C) A photograph of the anterior hairline scar at 2-year follow-up. Due to the trichophytic incision, the hair grew from the incision line and it could conceal the scars.
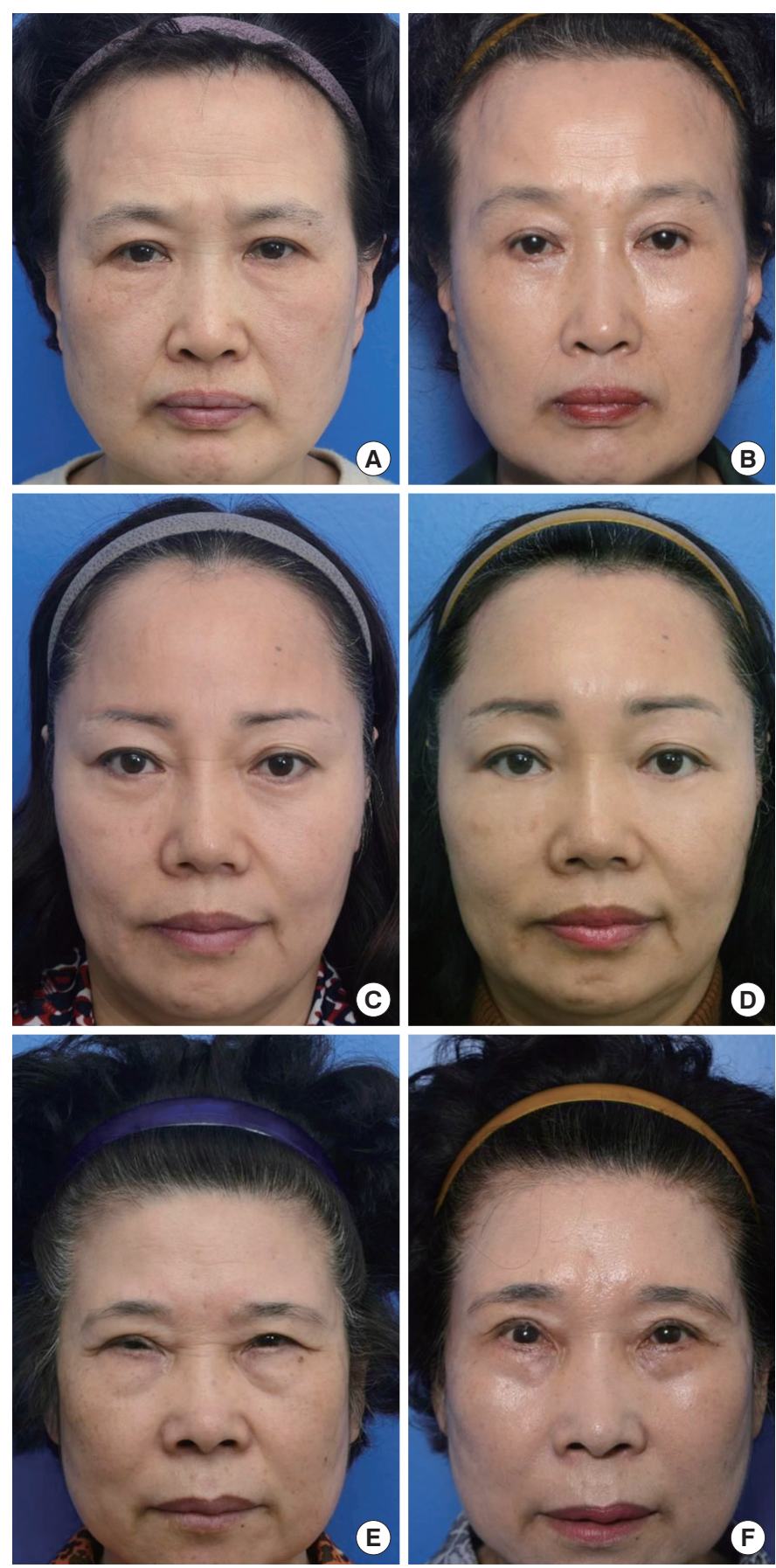

Fig. 5. (A) A preoperative clinical photograph of a 61-year-old female shows brow ptosis on both sides. (B) A postoperative 18-month follow-up photograph after anterior hairline forehead lift with lower blepharoplasty. The hair grew from the incision line and it could conceal the scars. (C) A preoperative clinical photograph of a 52-yearold female patient with high hairlines and brow ptosis on both sides. (D) A photograph at postoperative 8 months after an anterior hairline forehead lift with lower blepharoplasty. (E) A preoperative clinical photograph of a 68-year-old female with a drowsy appearance. (F) A photograph at postoperative 6 months after an anterior hairline forehead lift with upper and lower blepharoplasty with ptosis correction. 
phytic anterior hairline forehead lift with lower blepharoplasty. At postoperative 8 months, the photograph showed satisfying results (Fig. 5C and 5D). A 68-year-old female underwent an endoscopeassisted trichophytic anterior hairline forehead lift with upper and lower blepharoplasty with ptosis correction. At postoperative 6 months, the photograph showed satisfying results (Fig. 5E and 5F).

\section{DISCUSSION}

The level of an eyebrow is determined by the balance of the involved muscles. Aesthetically favorable brows lie at, or slightly above, the supraorbital rim. The lateral end should be slightly higher than the medial, peaking at the lateral one-third. In the aging process, the medial two-thirds of the eyebrow rises gradually by the action of the frontalis muscle. The lateral portion of the brow is sensitive to changes in age, because the frontalis muscle action is attenuated at the lateral side. Brow ptosis leads to an aesthetically displeasing 'sleepy' look and deep furrows on the forehead.

The brow lift plays an essential role for upper face rejuvenation [8]. Numerous techniques have been developed since Passot [9] first introduced their results 100 years ago. Among various options, the open coronal approach, anterior hairline approach, and endoscopic approach are the most popular methods for brow lift. The open coronal approach had been considered the gold standard. Many plastic surgeons still consider it the most effective method. The excellent surgical exposure makes it easier to manipulate the soft tissues around the brow. The results are enduring and stable. But a visible scar on the scalp and widening of the forehead are inevitable with this technique [2].

To minimize the disadvantages of the open coronal approach in a high-hairline patient, the anterior hairline approach has been used. It was introduced by Hunt [10] in 1926. It offers good surgical exposure and can lower the hairline. But it carries the disadvantages of visible scars along the anterior hairline and the risk of scalp denervation [3,4]. In 1992, Isse [11] and Vasconez [12] introduced the endoscopic brow lift. It offers good surgical exposure with magnification through small incisions. It carries less risk of scalp denervation. The principal disadvantages are the long learning curve and the inadequate fixations for the forehead musculature $[2,3]$.

We combined the two most frequently used methods-the anterior hairline approach and the endoscopic brow lift. With the help of an endoscope, the operator was able to obtain good surgical exposure and lower the risk of scalp denervation. Additionally, the incision scars could be camouflaged by the trichophytic incision, which was first introduced by Marzola [6] in 2005.

Our novel method has several advantages. First, a surgeon could minimize the risk of scalp denervation by direct visualization and preservation of the deep branches of the supraorbital nerve. Alth- ough 2 patients had temporary paresthesia, there were no permanent sensory problems. Second, the scar along the hairline could be hidden by the trichophytic incision. Third, this technique could adjust an unattractive hairline to conform to a more aesthetically acceptable forehead height, which is thought to range from $4.5 \mathrm{~cm}$ to $5.5 \mathrm{~cm}$ [13]. The operator could raise the low hairline as well as lower the high hairline.

Based on our results, we think our technique could be a safe and effective treatment option for brow ptosis patients with high hairlines.

\section{PATIENT CONSENT}

Patients provided written consent for the use of their images.

\section{REFERENCES}

1. Paul MD. The evolution of the brow lift in aesthetic plastic surgery. Plast Reconstr Surg 2001;108:1409-24.

2. Warren RJ. Forehead rejuvenation. In: Neligan PC, editor. Plastic surgery. 3rd ed. Philadelphia, PA: Saunders; 2012. p. 93-107.

3. Romo T, 3rd, Zoumalan RA, Rafii BY. Current concepts in the management of the aging forehead in facial plastic surgery. Curr Opin Otolaryngol Head Neck Surg 2010;18:272-7.

4. Nahai FR. The varied options in brow lifting. Clin Plast Surg 2013;40: 101-4.

5. Ramirez AL, Ende KH, Kabaker SS. Correction of the high female hairline. Arch Facial Plast Surg 2009;11:84-90.

6. Marzola M. Trichophytic closure of the donor area. Hair Transplant Forum Int 2005;15:113-6.

7. Singer AJ, Arora B, Dagum A, et al. Development and validation of a novel scar evaluation scale. Plast Reconstr Surg 2007;120:1892-7.

8. Guyuron B, Lee M. A reappraisal of surgical techniques and efficacy in forehead rejuvenation. Plast Reconstr Surg 2014;134:426-35.

9. Passot R. La chirurgie esthétique des rides du visage. Presse Med 1919; 27:258-60.

10. Hunt HL. Plastic surgery of the head, face, and neck. Philadelphia, PA: Lea \& Febiger; 1926.

11. Isse NG. Endoscopic forehead lift. The Annual Meeting of the Los Angeles County Society of Plastic Surgeons; 1992 Sep 25; Washington, DC.

12. Vasconez LO. The use of the endoscope in brow lifting. The Annual Meeting of the American Society of Plastic and Reconstructive Surgeons; 1992 Sep 25; Washington, DC.

13. Farkas LG, Eiben OG, Sivkov S, et al. Anthropometric measurements of the facial framework in adulthood: age-related changes in eight age categories in 600 healthy white North Americans of European ancestry from 16 to 90 years of age. J Craniofac Surg 2004;15:288-98. 